\title{
LA INVESTIGACIÓN GEOLÓGICA EN LA COMISIÓN CIENTÍFICA DE MÉXICO
}

\author{
Luz Fernanda Azuela ${ }^{1}$ \\ Instituto de Geografía \\ Universidad Nacional Autónoma de México \\ lazuelab@yahoo.com.mx
}

Recibido: 18 septiembre 2014; Aceptado: 14 julio 2015.

Cómo citar este artículo/Citation: Azuela, Luz Fernanda (2015), "La investigación geológica en la comisión científica de México", Asclepio, 67 (2): p101. doi: http://dx.doi.org/10.3989/asclepio.2015.19

RESUMEN: Durante el Imperio de Maximiliano (1864-1867) la ciencia mexicana experimentó cambios significativos en sus perspectivas generales y su enfoque metodológico, gracias a la participación de la comunidad científica local en los diferentes proyectos de investigación que se organizaron. Por un lado destacaron las propuestas del propio Emperador, quien impulsó la práctica científica local mediante el apoyo a los proyectos en curso, al tiempo que promovía nuevas empresas de investigación. Simultáneamente, Napoleón III ordenó al Instituto de Francia la organización de una expedición científica con el objeto de estudiar diferentes aspectos del país. Entre los objetivos de la expedición francesa destacó la investigación geológica, que se abordó tomando como antecedentes los estudios efectuados por numerosos viajeros europeos en los años precedentes. Este trabajo expondrá los principales rasgos de estos estudios y analizará los objetivos y alcances de la expedición durante su breve estancia en territorio mexicano.

PALABRAS CLAVE: Geología; México; Viajeros; Segundo Imperio; Francia.

\section{THE GEOLOGICAL RESEARCH IN THE SCIENTIFIC COMMISSION OF MÉXICO}

ABSTRACT: During Maximilian's Empire, Mexican science experimented changes both in its general perspectives and its methodological outlook, due to scientific interaction among the local scientific community and foreign scientists. Maximilian encouraged local scientists to continue working in their ongoing projects, and invited others to participate in new scientific endeavors. Simultaneously, Napoleon III instructed the French Institute to organize a scientific expedition in order to study different aspects of the new Empire. Geological research stood out among the goals of the French expedition, and it was initiated taking into consideration several geological studies made by European travelers during the first half of the century. This paper will describe the main features of such investigations and will analyze the expedition's goals and accomplishments during its brief sojourn in Mexico.

KEY WORDS: Geology; Mexico; Foreign travelers; Second Empire; France.

Copyright: () 2015 CSIC. Este es un artículo de acceso abierto distribuido bajo los términos de la licencia Creative Commons Attribution-Non Commercial (by-nc) Spain 3.0. 


\section{LA INVESTIGACIÓN GEOLÓGICA EN MÉXICO COMO OBJETIVO LOCAL Y FORÁNEO}

La primera mitad del siglo XIX mexicano fue un período de inestabilidad continua debido a las pugnas entre los diversos grupos políticos, que frecuentemente condujeron a la guerra civil, así como al asedio de las potencias intervencionistas. La fragilidad del estado nacional facilitó la pérdida de casi la mitad del territorio durante la guerra con los Estados Unidos (1847-1848) y desembocó años después en la intervención francesa, que culminó con la imposición del imperio de Maximiliano de Habsburgo (1864-1867). Durante su breve gestión, Napoleón III ordenó al Instituto de Francia la organización de una expedición científica con el objeto de estudiar diferentes aspectos del país, entre los que destacó la investigación geológica, objeto de este ensayo.

Pese a las dificultades políticas y económicas que agobiaron al país en los años precedentes, los intelectuales mexicanos habían encontrado el espacio para desarrollar investigaciones científicas, que para el caso de la geología se explican en el artículo de Lucero Morelos de este mismo volumen. Baste aquí señalar, que estuvieron vinculadas con el reconocimiento territorial y la industria minera, y que fueron promovidas por el gobierno y los particulares con diversos fines. Simultáneamente, un numeroso contingente de viajeros europeos y norteamericanos habían recorrido el país con objetivos relacionados con las empresas mineras y colonizadoras que se establecieron después de la independencia, dejando testimonio de sus investigaciones en numerosos libros y artículos científicos, que fueron objeto de traducciones y reimpresiones en diferentes países. De esta manera se dieron a conocer en el extranjero algunas características de la constitución geológica y la riqueza natural de México, que contribuyeron a suscitar el interés de las naciones extranjeras en el potencial extractivo del país.

Fue así que durante el Imperio de Maximiliano se valoraran los estudios realizados por «un pequeño número de observadores [extranjeros], entre los que [citaron] en primera línea, después de Alexandre von Humboldt, a Burkart, von Gerolt y Berghes» (SainteClaire Deville, 1865, p. 37), como la base científica para el desarrollo de las investigaciones que se proyectaron en esos años. La importancia que se confirió a dichas investigaciones en detrimento de la que se había efectuado localmente, implicaba desconfianza respecto a su «precisión científica» y su carácter «po- sitivo", que no dejaron de manifestar. Pero también es cierto que desconocían la calidad y extensión de los trabajos locales, en virtud de su restringida difusión en publicaciones exclusivamente nacionales, en contraste con la amplia circulación de los estudios europeos, que mencioné. En este sentido, el conocimiento del territorio mexicano del que disponían era escaso y se limitaba a las regiones descritas en las obras de los viajeros, entre los cuales destacaba como fuente primigenia la del barón Alexander von Humboldt.

Esta apreciación obliga a una breve referencia a los trabajos realizados por los viajeros europeos, tomado como punto de partida la expedición de Humboldt y su estancia en la capital de la Nueva España, que otros estudiosos han analizado a profundidad, por lo que me limitaré a exponer sus principales logros en relación con las ciencias de la Tierra:

Después de sus experiencias en el cono sur, Alejandro de Humboldt llegó al puerto de Acapulco el 23 de marzo de 1803. Se estableció en la Ciudad de México, en donde como menciona Moncada «el virrey Iturrigaray lo recibió y le abrió las puertas de oficinas y archivos, cerradas no sólo a otros extranjeros sino aún a los científicos novohispanos y peninsulares, y le facilitó un pasaporte que le permitió viajar por el reino sin ningún inconveniente» (Moncada, 2000, p. 34). Sus viejos compañeros de estudios de Frieberg, Andrés Manuel del Río, catedrático de Minería, y el propio Director del Seminario, Fausto de Elhúyar, pusieron a su disposición las instalaciones del Colegio para que realizara sus estudios.

Humboldt contó así con extraordinarias facilidades: información oficial privilegiada y colaboradores del más alto nivel, que le acompañaron en sus expediciones y le proporcionaron estudios, datos, colecciones naturalistas y mapas, con los que integró su Ensayo Político sobre el reino de la Nueva España (18071811) y su Viaje a las Regiones Equinocciales del Nuevo Continente (1807-1834).

En ambas obras aparecen las coordenadas geográficas de los lugares que visitó; se registran observaciones astronómicas y meteorológicas; se presentan mapas y secciones de los sistemas montañosos que recorrió, con información geológica inédita; y se hacen descripciones de fauna y flora, correlacionadas con el espacio geográfico en el que se ubican. El Ensayo Político incluye además, un estudio detallado sobre las minas mexicanas, apoyado en abundantes datos estadísticos y pormenores sobre la explotación minera. 
En relación con las ciencias de la Tierra, el viaje americano le proporcionó datos sobre las componentes del campo magnético terrestre, que posteriormente elaboraría; descubrió la corriente marina en la costa occidental de Sudamérica - llamada originalmente de Humboldt y hoy conocida como corriente del Perú-; fue pionero en los estudios que relacionan las regiones geográficas con la flora y fauna locales e hizo importantes contribuciones al desarrollo de la geología a partir de sus estudios sobre los temblores de tierra y los volcanes americanos, que aparecieron publicados en sus «Volcanes y cordilleras de Quito y México».

Respecto al último punto, fue invaluable su experiencia en México en donde presenció la actividad del Volcán Jorullo, de donde derivó sus conclusiones sobre el papel desempeñado por las fuerzas eruptivas en la historia y desarrollo de la corteza terrestre, que se consideraron decisivas para descartar definitivamente la hipótesis de los neptunistas.

También hizo contribuciones a la enseñanza de la geología en el Real Seminario de Minería, a través de su «Pasigrafía o Ensayo Geognóstico sobre el yacimiento de las rocas en los dos hemisferios», que incluyó Andrés Manuel del Río en su Tratado de Orictognosia (Río, 1832).

En lo que toca a la mineralogía, Humboldt dedicó todo un tomo de su Ensayo Político al estudio de los minerales mexicanos, tanto en lo que concierne a descripción física como en cuanto a los métodos de prospección, explotación, beneficio y amalgamación, así como a la organización del trabajo, la tecnología y los rendimientos de cada una de las minas.

Además del valor intrínseco de sus estudios, las obras de Humboldt promovieron el interés de científicos, exploradores e inversionistas, que viajaron a México después de la independencia y realizaron estudios de varias disciplinas científicas. En particular, la década de 1824-1834 vio desembarcar viajeros vinculados con compañías mineras que pretendían establecerse en nuestro país, especialmente ingleses, seguidos por los alemanes y los norteamericanos.

La explotación minera en la variada y poco conocida topografía mexicana, exigía la atención de técnicos y científicos que efectuaran estudios sobre la geología de las diversas localidades mineras y de la potencialidad económica de los yacimientos, que financiaron los propios empresarios.
Una vez en México, los empresarios y exploradores enfrentaron las peculiaridades naturales y sociales del país, que les prodigaron materiales para escribir artículos y libros sobre sus experiencias, que acompañaron de registros y descripciones naturalistas, así como de mapas regionales, que con el tiempo conformaron un cúmulo significativo de datos científicos sobre la naturaleza y la constitución geológica de México.

Entre las empresas que se establecieron en esos años destaca la Compañía Alemana-Americana de Minas (CAAM), en la que participaba la propia «familia real prusiana» y sus directivos, Wilhelm Stein y Friedrich von Gerolt, se eligieron entre los secretarios del Ministerio de Minería de Prusia. Stein fue nombrado «agente director de la compañía minera en México para que adquiriera minas y las explotara con la ayuda de otros alemanes», mientras que el ingeniero de minas Friedrich von Gerolt se integró al consejo directivo (Ward, 1981, p. 353). Todos los directivos de la empresa fueron individuos altamente capacitados en disciplinas relacionadas con la explotación minera, que realizaron investigaciones sobre el entorno natural en el que se establecieron y dejaron escritos de diversa índole y calidad científica: Stein, publicó en Heidelberg un artículo en el que se refiere a la "Mina de San Guillermo cerca de Perote» y se interesó por los meteoritos mexicanos, de los cuales habría tomado muestras que se analizaron en Europa (Enciso y Enciso, 1995, p. 51). J. C. Schmidt, quien sucedió a Stein, mantuvo una abundante correspondencia con sus compatriotas en la que comentó y corrigió los escritos de Humboldt, que calificó de «exageradamente positivos». Y Carl Koppe, escribió una obra que «intenta dar una visión global del país siguiendo el modelo de Humboldt», con el apoyo de fuentes mexicanas y extranjeras contemporáneas. Su valor parece haber radicado en su actualización de los datos estadísticos y políticos de México y la inclusión de parajes que Humboldt no visitó (Mentz, 1982, p. 73). Asimismo, algunos de ellos establecieron relaciones con la comunidad científica local y comenzaron a publicar en ambos lados del Atlántico los estudios científicos que emanaban de sus investigaciones.

En cuanto a las obras de mayor valor científico, la historiografía mexicana del XIX distingue las de Carl von Berghes y Friedrich von Gerolt ${ }^{2}$, como las primeras investigaciones post-humboldianas que contribuyeron al conocimiento de la geología de México. Destacan aquí las primicias de la cartografía geológica mexica- 
na, por su Carta geognóstica del Estado de México, en donde se ubicaron y caracterizaron los principales distritos mineros del antiguo estado de México (Crespo, 1903, p. 54; Ordóñez, 1946, p. 6; Cserna, 1990, p. 6) ${ }^{3}$. De acuerdo con de Cserna, «la versión original de este mapa con secciones fue publicada a colores en 1827 , mientras que su texto explicativo apareció en los Erdmann's Annalen der Erd Völker-und Staatenkunde, en Berlín, en 1835» (Cserna, 1990, p.6).

Von Gerolt fue más prolífico que su coautor de la Carta geognóstica, pues entre 1825 y 1834 publicó 8 artículos sobre México de temas como yacimientos de litargio, metalurgia, perfiles geognósticos, volcanes y estudios geológicos de los distritos mineros que incluían registros astronómicos, barométricos y mineralógicos (Aguilar y Santillán, 1898, pp. 92-93). Su impacto fue considerable, ya que con excepción del que publicó en el Registro Trimestre de México, sus artículos, cartas y perfiles, aparecieron en dos o más revistas de editoriales europeas y americanas (Berlín, París, Heidelberg, Sttutgart, Düsseldorf, Bonn, Nueva York y México).

En lo que concierne a las relaciones de los alemanes con la comunidad científica local, von Gerolt figura entre los miembros correspondientes de la Sociedad Mexicana de Geografía y Estadística desde 1838 (Alamán, 1947, pp. 104-106). Carl von Berghes, por su parte, mantuvo una colaboración científica importante con el mineralogista mexicano José Ma. de Bustamante (m. 1834), de la que da testimonio la Descripción de la Serranía de Zacatecas, que Bergues completó con observaciones y comentarios, así como con «planos, perfiles y vistas» que trazó él mismo, para publicarla en 1834 bajo la rúbrica de Bustamante y su propia colaboración (Bustamante, 1834).

Otro beneficiario de los trabajos de Bustamante fue el mineralogista Joseph Burkart (1798-1874), a quien se debe la obra de mayor trascendencia científica sobre el tema que nos ocupa, escrita luego de una década de investigaciones en el país - que efectuó mientras dirigía la mina de Tlalpujahua-. Se trata del libro Estancia y viajes en México en los años 1825 hasta $1834 . .$. , publicado en alemán en dos volúmenes (Burkart, 1836), con una dedicatoria al Barón de Humboldt y el propósito explícito de ampliar sus investigaciones de acuerdo con el programa que dejara delineado en el Ensayo Político del Reino de la Nueva España.

El trabajo refiere el intrincado y penosísimo viaje que emprendió Burkart entre San Blas y Tampico
(Burkart, 1836, vol. 2, pp. 169-170), cuya minuciosa descripción introdujo a la literatura científica el paisaje de la franja comprendida entre los paralelos $22^{\circ}$ y 23‥ Además ascendió al Nevado de Toluca y reconoció el Jorullo; visitó los basaltos de la Hacienda de Santa María Regla ${ }^{4}$ y buscó tenaz pero infructuosamente «la masa [meteórica] de Durango» mencionada por Humboldt. Todas sus descripciones incluyen tablas, mapas, cortes, mediciones, registros y estadísticas, que corrigieron y completaron la visión de Humboldt sobre la geografía y la naturaleza mexicanas. Y en lo que concierne a la geología, sus observaciones incluyen discusiones teóricas relacionadas con los caracteres mineralógicos y las condiciones estratigráficas de las rocas principales.

Pero la obra está lejos de limitarse a los campos de la mineralogía o la geología, ya que abarca estudios sobre los volcanes, los meteoritos, las fuentes termales, las ciudades, los distritos mineros y la arquitectura precolombina. En relación con las localidades que visitó, Burkart incluye comentarios de orden sociológico y etnográfico, a la vez que atiende las condiciones orográficas, hidrográficas y climatológicas. Así, la descripción naturalista de Burkart admite también al hombre: su aspecto físico, sus actividades, sus costumbres, sus relaciones sociales, sus enfermedades, sus «vicios» y sus talentos particulares. Análogamente, su examen de las ciudades y poblaciones; de la desigualdad social y el orden político, conlleva la alusión al medio físico.

Estas características hicieron que la obra de Burkart tuviera un impacto de consideración en los círculos intelectuales, empresariales y políticos. Sus investigaciones fueron ampliamente difundidas en México y en Europa fue leído y discutido por naturalistas, geógrafos, geólogos y mineralogistas. En particular, durante la Intervención Francesa su libro fue evaluado por el geógrafo Louis Vivien de Saint Martín (18021897), quien lo equiparó con la obra de Humboldt y atribuyó a Burkart «los más grandes servicios para el estudio científico de los territorios mexicanos y el perfeccionamiento de su carta» (Vivien de Saint Martín, 1865, p. 250).

Esta apreciación resume de alguna manera la opinión que se tenía en Europa sobre el estado de la investigación geológica en México, en la que se valoraban especialmente los estudios de los viajeros, mismos que recibieron una buena acogida a nivel local por sus contribuciones al estudio del territorio mexicano 5 . 


\section{EL PROGRESO DE LA GEOLOGÍA EN EL FRAGOR DE LAS GUERRAS}

Mientras los peritos y viajeros ultramarinos exploraban nuestro país, el gobierno mexicano puso en marcha varios proyectos que involucraron estudios geográficos, geológicos y mineralógicos. Destacan los trabajos del Istmo de Tehuantepec (1823-1826), de los alrededores de la Ciudad de México (1825) y de la frontera noreste de México y los Estados Unidos (1827-1831), que tuvieron por objeto "observar los rasgos naturales [de cada región]; obtener información geográfica y apuntar datos exactos para los mapas» (Mendoza, 2000, p. 92). De las tres, la del Istmo de Tehuantepec se convertiría con el paso de los años, en una de las regiones mejor estudiadas del país, por su presumible potencial para la comunicación interoceánica. De la misma manera que el problema del desagüe en la Ciudad de México dio lugar al énfasis en el estudio de la Cuenca.

Entretanto, algunos gobiernos del interior efectuaron estudios regionales sobre la configuración del territorio y sus recursos naturales. Pero cada vez se hacía más evidente la necesidad de contar con investigaciones de carácter general que sirvieran como fundamento para la planeación a largo plazo y la organización de la República. Así surgió el proyecto de fundar una institución con los objetivos de construir la Carta de la República y levantar la Estadística Nacional y en 1833 se creó Instituto Nacional de Geografía y Estadística - actual Sociedad Mexicana de Geografía y Estadística $(\mathrm{SMGE})^{6}$ - , en donde se agrupó la comunidad científica e intelectual del país. De ahí que desde los primeros números de su Boletín aparecieran artículos relacionados con el estudio de los diferentes tópicos que iban constituyendo el saber geológico, como disciplina científica.

Las actividades de la SMGE fueron afectadas por la inestabilidad política, aunque nunca se suspendieron, ni siquiera durante la guerra con los Estados Unidos (1846-1848), que contra todo pronóstico, supuso grandes adelantos en la geología. Durante esos años se transformó en una Comisión de Estadística Militar, que continuó con las tareas de reconocimiento y cartografía, en las que se incluyeron algunos estudios geológicos.

Como es sabido, el conflicto bélico desembocó en una considerable pérdida territorial, que incluía Nuevo México y California, que sumado al de Texas - recientemente independizado y anexado a los Estados Unidos-, constituían más de la mitad del territorio mexicano ${ }^{7}$.
La guerra concluyó con la firma del Tratado de paz de Guadalupe-Hidalgo, el 2 de febrero de 1848, que ordenó la conformación de sendas Comisiones de Límites para efectuar los trabajos de delimitación en el campo (1849-1855). Las tareas se llevaron a cabo en condiciones adversas para los mexicanos, ya que sus ingenieros contaban con un pobre instrumental, con frecuencia en malas condiciones, que contrastaba notablemente con el equipamiento científico de los estadounidenses (Tamayo, 2000, pp. 69-70). De ahí que la Comisión mexicana se limitara a realizar trabajos cartográficos y topográficos, que aparecieron en sus informes y cartas. También se apuntaron ahí algunas notas sobre la configuración del terreno, como «los cursos de los ríos, los arroyos y pantanos notables, así como de la dirección general de [los] lomeríos y los cerros aislados...» (Tamayo, 2001, p. 105). Un cúmulo valioso de información, que permaneció inédito debido a las prioridades del Estado en aquellos años de inestabilidad y desgobierno.

En contraste, los comisionados estadounidenses acopiaran suficiente material para publicar una amplia bibliografía sobre sus observaciones físicas de la región fronteriza, que sólo en relación con Nuevo México sumaron un total de 19 obras publicadas que contienen descripciones y estudios geológicos de la región fronteriza (ACSM-1, pp. 266-275).

Los trabajos más valiosos se concentraron en el Levantamiento Emory (1857), que incluyó el estudio geológico de una amplia región situada, al norte y al sur de la nueva línea divisoria. A juicio de Cserna este estudio hizo "contribuciones importantes al conocimiento de la estratigrafía, la paleontología y la petrografía de las partes septentrionales de México» y constituye el primer estudio geológico realizado en el país estadounidense que representa una aportación al patrimonio científico del mundo (De Cserna, 1990, pp. 8-9).

En el nivel local, incluso en los peores años el interés en el desarrollo científico de México se mantuvo a contracorriente. En el terreno de la práctica destaca la formación de una Comisión para levantar la Carta Geográfica y Topográfica del Valle de México (1857), con el objetivo de alcanzar un conocimiento más completo sobre la ciudad de México y sus alrededores. Las dificultades políticas impidieron que los trabajos alcanzaran la totalidad de los objetivos, pero se publicaron varios trabajos parciales, entre los que destaca la Memoria para la carta hidrográfica del Valle de México, que consigna algunos datos geológicos del área (Orozco y Berra, 1862, pp. 337-512). 
Hubo también en estos años viajeros que realizaron investigaciones científicas en nuestro país, entre los que destaca el prolífico naturalista suizo Henri de Saussure, quien examinó las solfataras de la Sierra de San Andrés en el Estado de Michoacán y construyó la Carta de México representando el Valle de Anáhuac y su vertiente oriental (De Cserna, 1990, p. 10; Orozco y Berra, 1881). Publicó sus datos y observaciones en los libros Mémoires pour servir a l'histoire naturelle du Mexique, des Antilles et des États-Unis -1858- y Coup d'oeil sur l'hydrologie du Mexique (1862).

Otro viajero que hizo contribuciones a la geología mexicana, fue William M. Gabb, quien incursionó en territorio mexicano como miembro de la California Geological Survey, en los años sesenta del siglo XIX, publicando un trabajo sobre los recursos minerales de Baja California, que tituló "Lower California» (1868). También escribió sobre sus fósiles cretácicos y terciarios (1869), que luego completó con los de Sonora y Chihuahua (1872), que aparecieron por separado, en la serie de la Geological Survey, bajo los subtítulos de "Geology» y "Paleontology.» Los demás se publicaron en los Proceedings of the Academy of Natural Sciences of Philadelphia. (Aguilar y Santillán, 1898, p. 86; Torres, 2012, pp. 115-125) ${ }^{8}$.

Entretanto, el gobierno liberal bajo el liderazgo del Presidente Juárez (1861-1863), trataba de impulsar diversos proyectos que impulsarían el desarrollo científico del país ${ }^{9}$, que se vieron truncados por la invasión de las tropas francesas que apoyaron el ascenso de Maximiliano de Habsburgo como Emperador y la subsecuente guerra anti-intervencionista.

\section{LA GEOLOGÍA DURANTE EL SEGUNDO IMPERIO}

Cuando Maximiliano asumió el poder reconoció la necesidad de contar con una comunidad científica activa, como elemento indispensable para impulsar la modernización de su nueva patria. En consecuencia, el Imperio recurrió a la plataforma de las escuelas locales de Medicina y Minería, así como en la Sociedad Mexicana de Geografía y Estadística, a las que brindó su incondicional apoyo a cambio de su participación en sus proyectos. La respuesta de los intelectuales fue de asentimiento casi unánime, con la excepción de aquellos que tomaron las armas contra los franceses. En cambio algunos consideraron el establecimiento del Imperio como fait accompli -cuyo futuro o duración era difícil de prever- y acataron el nuevo orden sin mayor resistencia. Otros lo hicieron con entusiasmo debido al clima progresista que dominaba en la capital del país por el inesperado liberalismo de Maximiliano, que provocó la simpatía de los intelectuales moderados con las avanzadas propuestas del Emperador. Así, los que estuvieron en una posición directiva - como el geógrafo e historiador liberal Manuel Orozco y Berra en el Ministerio de Fomento-, aprovecharon la disposición del monarca para mantener y promover los proyectos republicanos de modernización del país. Como consecuencia, Maximiliano promovió con relativo éxito una serie de acciones de carácter científico, en las que se manifestaron los intereses de diferentes redes:

Unas de estas acciones consistieron en la revitalización de viejos proyectos locales como la Comisión del Valle de México y el proyecto del desagüe (Espinosa, 1902, p. 309), la Academia de Medicina y el Observatorio Astronómico y Meteorológico; otras, fueron propuestas novedosas como la formación de la Carta Geológica de los distritos minerales, o la localización geográfica y la descripción de monumentos arqueológicos. Además hubo lugar para las aficiones personales del monarca, cuyo proyecto personal fue la habilitación del Museo Público de Historia Natural, Arqueología e Historia ${ }^{10}$, que encargó al entomólogo Dominich Billimeck (Azuela y Vega y Ortega, 2011).

Al mismo tiempo que se delinearon los proyectos científicos de Maximiliano, el Mariscal Bazaine, que encabezaba el ejército intervencionista, quiso aprovechar los talentos científicos bajo su mando para recabar información y desarrollar estudios sobre México. Simultáneamente, Napoleón III -que no quería ser menos que Carlos X en Grecia y Luis Felipe en Argelia - ordenó la conformación de la Commission Scientifique du Mexique (1864-1867) bajo la dirección del Instituto de Francia, que se abordará en las siguientes páginas ${ }^{11}$.

El proyecto de la expedición francesa tenía claros objetivos expansionistas, que el emperador francés no se cuidó de ocultar, como puede observarse en la transcripción que hizo el ministro Victor Duruy de sus intenciones, en una carta dirigida al Presidente de la Sociedad Mexicana de Geografía y Estadística:

El Emperador, que nunca deseó llevar a cabo una conquista por las armas, tiene la noble ambición de conquistar su grandioso país para la ciencia. Nuestros sabios van a marchar una vez más sobre las huellas de nuestros soldados, pero con mayor fortuna que sus predecesores del Instituto de Egipto. Pues ahora 
encontrarán a su llegada, numerosos trabajos ya desarrollados y sociedades de sabios que están organizadas desde hace tiempo ${ }^{12}$.

Se trataba, como es claro, de analizar científicamente las condiciones del país en todos sus aspectos para lograr un eficiente control político y promover la efectiva explotación de los recursos naturales. Por ello, el decreto de conformación de la CSM estableció como prioridad los estudios «sobre la geografía; la constitución geológica y mineralógica del país; la descripción de las especies animales y vegetales; el estudio de los fenómenos atmosféricos y de la constitución médica; el de las diversas razas, sus monumentos [y] su historia» (ACSM-1, p. 16). Temas, que se habían mantenido en la agenda de todos los gobiernos del país, por lo que abundaban los estudios locales desde el siglo XVI y existían avances sustantivos para las tareas que se emprenderían. Los franceses lo reconocieron cabalmente, aunque como señalé, no se guardaron de expresar desconfianza respecto a su «precisión científica» y su carácter "positivo».

La Commission se dividió en cuatro Comités de acuerdo con la especialidad de los estudios e inscribió la geología en el de Ciencias Naturales y Médicas. El Comité quedó integrado por 5 miembros del Instituto de Francia: el naturalista Henri Milne Edwards (18001885) ${ }^{13}$; el botánico Joseph Decaisne (1807-1882) ${ }^{14}$; el naturalista y antropólogo Jean-Louis-Armand de Quatrefages (1810-1892) ; $^{15}$ el geólogo Charles Sainte-Claire Deville (1814-1876) ${ }^{16}$; y el Barón Félix Hippolyte Larrey (1808-1895), cirujano militar y hombre político ${ }^{17}$.

En las "Instrucciones Sumarias» que redactó, el Comité definió su objeto de estudio en los siguientes términos:

Desde el punto de vista de las ciencias naturales, la exploración científica de una región cualquiera comprende el estudio de las razas humanas que la han habitado desde el pasado hasta la actualidad; la descripción de las especies animales y vegetales actualmente vivas [y] de las extintas; la búsqueda de los elementos de la constitución del suelo; [y] la observación de los fenómenos geológicos que puede aún escenificar (Comité des Sciences Naturelles et Médicales, 1865, p. 19).

Aunque el Comité reconoció la posibilidad de estudiar simultáneamente los aspectos «prácticos» de la naturaleza mexicana, advirtió que "los viajeros y los corresponsales de [la Commission] deberían [mantener como prioridad] el estudio puramente 'científico' del país». (Comité des Sciences Naturelles et Médica- les, 1865, p. 20) El texto prosigue con los lineamientos generales que orientarían las expediciones y enseguida aparecen los instructivos de cada disciplina, que redactó por separado el especialista.

El instructivo de geología y mineralogía fue elaborado por Charles Saint-Claire Deville, quien inició sus disposiciones indicando que su meta sería la construcción de la carta geológica de México. Aunque advirtió que como ésta representaba «el resumen gráfico» de la minuciosa exploración geológica del territorio, «la carta [...], sería el resultado de largos y perseverantes esfuerzos». Sobre todo, porque dependería del "conocimiento suficientemente exacto de la topografía local» (Sainte-Claire Deville, 1865-1867, p. 37) ${ }^{18}$. Es decir, se trataba de un proyecto para el mediano plazo.

Entretanto, era preciso recoger «los datos generales sobre la constitución geognóstica del nuevo imperio mexicano, o si se quiere, ampliar y completar los que se deben a un pequeño número de observadores, entre los que habría que citar en primera línea, después de Alexandre von Humboldt, a Burkart, de Gerolt y de Berghes ${ }^{19}$. Y ordenó que sólo «en los casos particulares y para las regiones que presentaran un interés capital, ya fuera desde el punto de vista de los fenómenos eruptivos, o desde el punto de vista de la geología estratigráfica o palentológica», los expedicionarios podrían recurrir «al apoyo de los trabajos locales». En estos casos, «convenientemente elegidos, agregó, se daría un gran servicio a la ciencia con la elaboración de monografías sobre las semejanzas y anomalías que se puedan observar entre las formaciones eruptivas o sedimentarias de América Central (sic) y aquéllas de Europa o de Norteamérica».

El geólogo francés manifestó su interés en «el estudio químico de las emanaciones volcánicas y la descripción de los restos orgánicos de los terrenos estratificados». Y «en el mismo sentido, señaló, debe concederse mucha atención a una mina igualmente fecunda, pues ambas cosas juntas sólo han aflorado hasta hoy, en [el territorio de] México ${ }^{20}$.

El «Instructivo» prosigue con la descripción de los diferentes tipos de terreno, que se habían identificado y la enumeración de los objetos de estudio que debían atender los expedicionarios. Destacó aquí la identificación y estudio de las vetas metalíferas, "sin ninguna duda, la mayor riqueza natural de México»; de los veneros de aguas minerales y las «chimeneas volcánicas que forman los puntos culminantes del Nuevo Mundo»; demandó la formación de colecciones de minerales, como paso previo e indispensable 
para el estudio litológico; y enfatizó el interés en «recoger» meteoritos, o "por lo menos muestras» para su análisis en el laboratorio. En todos los casos los reportes se debían acompañar de dibujos y fotografías cuando fuera posible.

A continuación Sainte-Claire expone las «recomendaciones generales», no sin antes aconsejar la "colecta de todos los datos existentes en el país, impresos o manuscritos [...] sobre la geografía, la topografía, la geología y la mineralogía de México». (Comentario que indica la ineludible validación de las capacidades científicas locales, que previamente había despreciado).

Entre las recomendaciones destaca el dibujo preciso de las formaciones exploradas; la elaboración de esbozos de los cortes geológicos; el registro de observaciones barométricas y altimétricas con instrumentos específicos; la identificación in situ de las muestras minerales y paleontológicas, si fuera posible; y su etiquetación con todos los datos del lugar donde se encontraron.

En lo que toca a las minas, el geólogo aconsejó el acopio de los levantamientos de las minas ya explotadas, que efectuarían «los ingenieros locales». Encomendó el examen minucioso del orden y la sucesión de las substancias de la veta y la colección de muestras, "especialmente de las especies cristalizadas» ${ }^{21}$. De las aguas minerales pidió su localización y análisis químico, igual que respecto a los volcanes, cuyas emanaciones había que determinar, lo mismo que sus temperaturas. Y reiteró su interés en los meteoritos.

El «Instructivo» incluye detalles sobre el uso de los instrumentos adecuados y consejos para situaciones específicas, que no cabe enumerar. Aunque sí vale la pena extenderse en el comentario sobre la trascendencia de la expedición sobre el desarrollo ulterior de la geología en México, pues el "Instructivo» simbolizaba la difusión de la metodología de frontera para la investigación geológica.

De hecho, la geología fue justamente el área de investigación en la que se efectuaron «los mejores y más amplios trabajos» de la CSM (Maldonado-Koerdell, 1965, p. 172). Desde luego, el crédito corresponde a los expedicionarios: los mineralogistas y geólogos E. Guillemin-Tarayre, Auguste Dollfus, E. de Montserrat y P. Pavie, quienes exploraron una amplia región del país e hicieron contribuciones significativas al conocimiento de la conformación geológica de los distritos mineros y a la determinación de la edad de algunas formaciones mexicanas.

\section{ALGUNOS RESULTADOS DE LA COMMISSION SCIEN- TIFIQUE DU MEXIQUE}

Tal vez el resultado más importante de los geólogos franceses fue su desempeño como instructores de los científicos locales que se sumaron a la expedición para llevar a cabo trabajos de campo y de gabinete. Algunos fueron nombrados corresponsales en México por el Ministerio de la Instrucción Pública francés (Antonio del Castillo, Antonio García y Cubas, Francisco Jiménez, Manuel Orozco y Berra) y otros como Leopoldo Río de la Loza o Ramón Almaraz, simplemente figuraron entre los colaboradores.

En lo que concierne al alcance geográfico de sus investigaciones geológicas, éstas sólo pudieron efectuarse en áreas restringidas en virtud de la extensión de la guerra entre los ejércitos imperiales y republicanos. Sin embargo, las regiones que alcanzaron a explorar incrementaron el reconocimiento territorial y los estudios geológicos del país, dando lugar a numerosos trabajos científicos. Entre los más valorados por los geólogos mexicanos se puede mencionar el Coup d'Oeil sur la Topographie et la Géologie du Mexique et de l'Amérique Centrale de Virlet d'Aoust, que Aguilera destaca por la descripción y fechamiento del "sistema de Anáhuac»; la identificación de los distintos tipos de terreno y de minerales del país. Pone en relevancia sus aportaciones al estudio del metamorfismo y sus trabajos sobre los terrenos de origen meteórico (Aguilera, 1896, pp. 59-60). Carron de Fleury publicó unas «Notas geológicas y estadísticas de Sonora y la Baja California...» (Carron de Fleury, 1869, pp. 44-52 y 112-118.); Antonio del Castillo hizo un "Cuadro de la mineralogía mexicana", en donde ordenó las especies minerales de acuerdo con su composición química y cristalización, "con el arreglo al sistema del profesor Dana» y efectuó un estudio sobre el fierro meteórico de Yanhuitlán (Castillo, 1864-1, pp. 564-571 y 1864-2, pp. 661-665). Y Pierre Laur escribió un tratado de 300 páginas sobre la metalurgia de la plata mexicana, que apareció en los Annales des Mines de París en $1871 .^{22}$

Los expedicionarios de la Commission, por su parte, dieron a la imprenta numerosas memorias geológicas, de acuerdo con los lineamientos del Instructivo:

Sobre los volcanes, Auguste Dollfus y Eugène de Montserrat firmaron un estudio sobre el Nevado de Toluca y construyeron los cortes geológicos y el plano del cráter, mismo que acompañaron con un croquis geológico y topográfico de los alrededores de Toluca; y también fueron coautores de un trabajo sobre el Volcán de Colima. En colaboración con P. Pavie, am- 
bos autores efectuaron el estudio del Popocatépetl y trazaron el corte geológico de la cima (ACSM-3, pp. 29-35, 43-55 y ACSM-2, pp. 187-201). Posteriormente los dos primeros hicieron un «Viaje geológico a las repúblicas de Guatemala y El Salvador», en cuyo reporte se refirieron brevemente al volcán de Tacaná, Chiapas e hicieron algunas anotaciones "sobre temblores y erupciones volcánicas» ${ }^{23}$.

La región que exploraron incluye los estados de México, Puebla y Veracruz, desde donde se desplazaron al sureste para la expedición a Centroamérica. De acuerdo con las instrucciones de Sainte-Claire, tuvieron el cuidado de anotar minuciosamente sus observaciones y trazar los cortes de sus travesías. De ahí resultaron artículos sobre el trayecto de Veracruz a México, del que trazaron un corte que «sigue el desarrollo de la línea quebrada que pasa por México, Puebla, Orizaba y Veracruz» (ACSM-2, pp. 124-127). También publicaron un estudio geológico a partir de los cortes de Naolinco a Huatusco, de Perote a Tehuacán y de ahí a Puebla, a los que se sumaron los cortes paralelos y transversales de la cordillera. Este trabajo incluye la carta geológica del distrito de Zomelahuacan y el plano de las aguas minerales de los alrededores de Puebla (ACSM-2, pp. 363-403).

Respecto a los distritos mineros, Dollfus y Monserrat publicaron un estudio sobre el de Sultepec, que contiene tres apartados. El primero se dedica a la geografía física (montañas, hidrografía y clima); en el segundo, titulado "Geología», se ocupa de la identificación de las formaciones geológicas, volcanes, aguas minerales "y emanaciones gaseosas». Y bajo la rúbrica de «Mineralogía y fábricas metalúrgicas», el último se refiere a los filones metalíferos y las haciendas de beneficio. El trabajo incluye 3 cortes geológicos de los derroteros de la expedición (ACSM-3, pp. 471-496).

El mineralogista Guillemin Tarayre, por su parte exploró el noroeste de México (Baja California y Sonora) y después de la caída del Imperio, parece haberse desplazado hacia los Estados Unidos, para integrar aquella región con los estudios geológicos y mineralógicos de la Alta California y Nevada. Entretanto, viajó a Sinaloa, Chihuahua, Durango, Zacatecas, San Luis Potosí, Guanajuato, Jalisco, Hidalgo y México.

Fue el más prolífico de los expedicionarios, ya que entre los reportes que envió al Instituto de Francia que se publicaron en los Archives-, destaca uno de 300 páginas sobre «la exploración mineralógica de las regiones mexicanas», en el que además de anotar los usuales datos sobre los distritos mineros, se ocupa de la temperatura comparativa de las aguas del Atlántico en las costas de América y Francia; incluye observaciones de asteroides y se refiere al meteorito de Casas Grandes $^{24}$

En 1870 publicó un libro sobre «La producción de metales preciosos en la América Septentrional», que completa con el mapa de «las dos Californias, Nevada y los territorios circunvecinos" y los cortes geológicos de California y Nevada, así como el de Baja California. Contiene asimismo, un perfil geológico del país entre los dos océanos», que trazó durante su travesía de San Blas a Veracruz.

En todos los casos, Guillemin tuvo el cuidado de proporcionar una visión de conjunto que no dejó fuera los datos históricos ni las descripciones del paisaje y los pobladores. Pero además, proporcionó información paleontológica, arqueológica y etnográfica de zonas que habían permanecido en el olvido - Casas Grandes, por ejemplo. Todo ello complementado con las indispensables estadísticas económicas de las minas y de la región circundante (véase figura 1).

Los estudios geológicos de los Archives... se completan con los trabajos de otros estudiosos como Laur, al que me referí anteriormente ${ }^{25}$; y con los que discuten los reportes de los expedicionarios, que firmaron Sainte-Claire Deville y Combes ${ }^{26}$.

En total, los trabajos de la CSM llegaron a completar 3 gruesos volúmenes en los que se incluyen las actas de las sesiones de trabajo; los reportes de los viajeros y estudios extensos como los que he mencionado. Otras investigaciones se dieron a la imprenta en la serie de la Mission Scientífique au Mexique et dans I'Amérique Centrale (1868), una elegante colección de grandes tomos en folio, profusamente ilustrada. Mientras que los militares publicaron sus estudios en una serie por separado, L'Expédition au Mexique, donde apareció la Carta del Imperio Mexicano del General Gustave Niox (Niox, 1873).

A la productividad bibliográfica habría que añadir las colecciones y especímenes que fueron a dar a París para enriquecer el patrimonio cultural de Francia. Tal vez el mejor indicador del volumen que acopiaron, fue el traslado del meteorito de Charcas - de $780 \mathrm{~kg}$ - que condujo Bazaine hasta el Museo de París. Una metáfora material de las transferencias científicas y culturales que se verificaron durante el malogrado imperio. 
Figura 1. Exploración geológica de la Commission Scientifique du Mexique

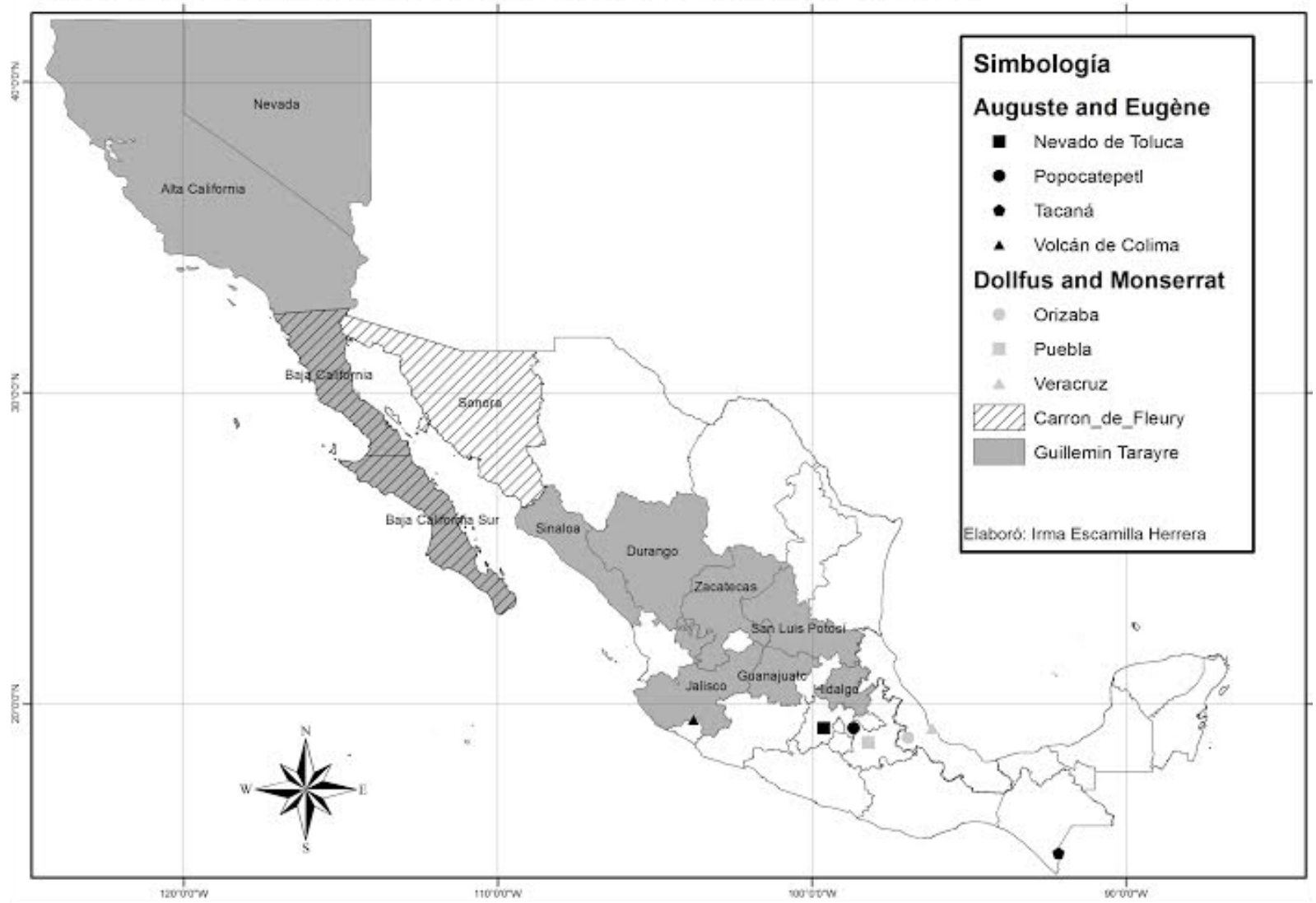

\section{NOTAS}

1 Esta investigación es parte del proyecto PAPIIT núm. IN 301113-RN 301113: "La Geografía y las ciencias naturales en algunas ciudades y regiones mexicanas, 1787-1940". Responsable Dra. Luz Fernanda Azuela, Instituto de GeografíaUNAM.

2 Friedrich von Gerolt nació en Linz, cerca del Rhin (c. 1800). Estudió en el Politécnico de París y luego defendió su patria en la guerra franco-prusiana. Emigró a Inglaterra (1824) y se incorporó a la CAAM. Fue consejero de la Cía. inglesa de Real del Monte. Regresó a Alemania en 1836, pero volvió a México un año después como Cónsul Plenipotenciario de Prusia, puesto en el que permaneció hasta 1846, cuando partió con el mismo cargo a los Estados Unidos. (Diccionario Porrúa de Historia, Biografía y Geografía de México, 1995, p. 1437)

3 El territorio del estado de México comprendía entonces partes de los estados de México, Guerrero, Hidalgo, Tlaxcala y Morelos.

4 Estos sitios aparecen reiteradamente en la literatura de viajes debido a la influencia de Humboldt, quien no sólo los describió maravillado, sino que enfatizó la importancia de efectuar otras investigaciones. De Santa María Regla realizó incluso dibujos, que otros repitieron después con la misma perspectiva. (Burkart, pp. 182-188 y p. 66)

5 Otro viajero que contribuyó al estudio de la geología mexicana fue el geógrafo y naturalista belga Henri Galeotti (Azuela, 2012).

6 El Instituto cambió dos veces de denominación y adquirió su nombre actual en 1850 .

7 En 1853 el Tratado de la Mesilla modificó parte de los límites establecidos en el anterior.

8 Sobre los pormenores de la expedición véase Torres, 2012.

9 Durante su gestión, el ingeniero geógrafo Francisco Díaz Covarrubias propuso la creación de un Observatorio Astronómico en la ciudad de México y el establecimiento de una red de observatorios meteorológicos en la República, con el fin de ampliar y sistematizar las observaciones en todo el país. 
10 El Museo Nacional de México se inauguró durante el gobierno de Guadalupe Victoria en 1825 y se mantuvo hasta el Segundo Imperio.

11 El decreto indica que el Ministerio de Instrucción Pública de Francia supervisaría la organización de los trabajos. (ACSM-1, p. 8).

12 La carta, fechada el 8 de febrero de 1864, está firmada por el Ministro de Instrucción Pública Victor Duruy (18111894), historiador y reformador de la enseñanza francesa (ACSM-1, p. 14)

13 El naturalista Milne Edwards fue director del Museo de Historia Natural y autor de las Leçons d'anatomie et de physiologie comparées.

14 Decaisne era miembro de l'Academie des Sciences (1847), a la que presidió en 1865 .

15 Quatrefages, miembro de l'Academie des Sciences (1852), defendió la teoría de la unidad de origen del hombre.

16 Sainte-Claire Deville, miembro de l'Academie des Sciences (1857) y director fundador del Observatoire de Montsouris. Estudió las propiedades del azufre y creó una teoría sobre los volcanes.

17 Larrey, profesor de Val de Grâce, Paris; miembro de la Academie Impériale de Medicine y del Consejo de Guerra.

18 Las siguientes citas están tomadas del mismo texto (pp. 3748) por lo que omitiré la referencia.

19 Curiosamente, durante el Imperio se reeditaron en Nueva York los trabajos geológico-cartográficos de Berghes y von

\section{BIBLIOGRAFÍA}

Aguilar y Santillán, R. (1898), “Bibliografía geológica y minera de la República Mexicana”, Boletín del Instituto Geológico de México, 10, pp. 1-159.

Aguilera, J. G. (1896), Bosquejo Geológico de México. México, Oficina Tipográfica de la Secretaría de Fomento.

Alamán, L. (1947), Obras, 4 vols. México, Editorial Jus.

Archives de la Commission Scientifique du Mexique (ACSM-1) (1865), Paris, Ministère de I'Instruction Publique, Imprimerie Impériale.

Archives de la Commission Scientifique du Mexique (ACSM-2) (1866), Paris, Ministère de I'Instruction Publique, Imprimerie Impériale.

Archives de la Commission Scientifique du Mexique (ACSM-3) (1867), Paris, Ministère de I'Instruction Publique, Imprimerie Impériale.
Gerolt, que se publicaran en Alemania en 1827. Fue el barón Friedrich von Egloffstein, quien se dio a la tarea de darlas a la imprenta, a través de la casa editorial Appleton y Cía. (Nueva York, 1864). Con ello, comenta de Cserna, fueron más fácilmente accesibles "al público interesado en este lado del Atlántico” (De Cserna, 1990, pp. 5-6).

20 Aquí el especialista se refiere a la riqueza del territorio tanto para la explotación de las minas como para el adelanto de la investigación geológica.

21 Para las minas hubo además un instructivo específico en el que se encareció la necesidad de formar una carta de las minas del país.

22 Esta obra, dice Aguilar, se convirtió en uno de los trabajos más importantes de su tiempo (Aguilar, 1898, pp. 133-134).

23 Este viaje se publicó en las memorias de la Mission Scientífique au Mexique et dans l'Amérique Centrale (1868).

24 Guillemin envió un total de 6 trabajos, el último fue el "Rapport à son Exc. M. Le Ministre de l'Instruction Publique sur l'exploration minéralogique des régions mexicaines", reeditado como "Exploration minéralogique des Régions Mexicaines suivie des Notes Archéologiques et Étnographiques" (ACSM-1, pp. 173-470).

25 Entre muchos otros están los estudios geológicos de la frontera norte de Jules Marcou (ACSM-2, pp. 74-80).

26 Sainte-Claire firmó 6 trabajos relativos a los reportes de los exploradores y Combes, publicó 3 (ACSM-1, 2 y 3).

Azuela, L. F. y R. Vega y Ortega (2011), "El Museo Público de Historia Natural, Arqueología e Historia (1865-1867)". En: L. F. Azuela y R. Vega y Ortega (Coord.), La geografía y las ciencias naturales en el siglo XIX mexicano, México, Universidad Nacional Autónoma de México, pp. 103-120.

Azuela, L. F. (2012), “El territorio mexicano en los estudios algunos viajeros del siglo XIX". En: L. F. Azuela y R. Vega y Ortega (Coord.), Naturaleza y territorio en la ciencia mexicana del siglo XIX, México, Universidad Nacional Autónoma de México, pp. 85-105.

Burkart, J. (1836), Aufenthalt und Reisen in Mexico in den Jahren 1825 bis 1834. Bemerkungen über Land, Produkte, Leben und Sitten der Einwohner und Beobachtungen aus dem Gebiete der Mineralogie, Geognosie, Bergbukunde, Meteorologie, Geographien, etc, 2 vols. Stuttgart, E. Schweixwerbart's Verlagshandlung.

Bustamante, J. M. (1834), Descripción de la Serranía de Zacatecas formada por... Aumentada y combinada con planos, 
perfiles y vistas trazadas en los años de 1829, 1830, 1831 y 1832 por C. De Berghes. México, Imprenta de Galván.

Carron de Fleury, S. E. L. (1869), “Notas geológicas y estadísticas de Sonora y la Baja California. Situación geográfica. Descripción física. Origen de la población actual", Boletín de la Sociedad Mexicana de Geografía y Estadística, vol. I, 2a época, pp. 44-52, pp. 112-118.

Castillo, A. (1864a), "Cuadro de la mineralogía mexicana", Boletín de la Sociedad Mexicana de Geografía y Estadística, vol. X, 1a época, pp. 564-571.

Castillo, A. (1864b), “Descripción de la masa de hierro meteórico de Yanhuitlan, recientemente traída a esta Capital y noticia y descripción de las masas de hierro meteórico, y de piedras meteóricas caídas en México", Boletín de la Sociedad Mexicana de Geografía y Estadística, vol. X, 1a época, pp. 661-665.

Crespo y Martínez, G. (1903), México. Industria Minera. Estudio de su evolución por..., para la grande obra México: su evolución social. México, Oficina Tipográfica de la Secretaría de Fomento.

Comité des Sciences Naturelles et Médicales (1865), "Instructions Sommaires". En: Archives de la Commission Scientifique du Mexique; vol. I, Paris, Ministère de I'Instruction Publique, Imprimerie Impériale.

De Cserna, Z. (1990), "La evolución de la geología en México (c. 1500-1929)", Revista del Instituto de Geología, vol. IX (1), pp. $1-20$.

Enciso, S. y C. Enciso (1995), "Bosquejo histórico de la mineralogía mexicana", Revista GEOMIMET, 196, pp. 48-55.

Espinosa, L. (1902), "Reseña histórica y técnica de las obras del desagüe del Valle de México, 1856-1900". En: Memoria histórica, técnica y administrativa de las obras del desagüe del Valle de México. 1449-1900, vol. I.,() México, Tipografía de la Oficina Impresora de Estampillas, pp. 276-431.

Garibay K., Angel María (dir. y pref.) (1995), Diccionario Porrúa de Historia, Biografía y Geografía de México, México, Porrúa.

Maldonado-Koerdell, M. (1965), "La obra de la Commission Scientifique du Mexique". En: A. Arnaiz y Freg, y C. Bataillon (eds.), La intervención francesa y el Imperio de Maximiliano cien años después, 1862-1962, México, Asociación Mexicana de Historiadores, Instituto Francés de América Latina, pp. 160-182.
Mendoza, H. (2000), "Las opciones geográficas al inicio del México independiente“. En: H. Mendoza (coord.), México a través de los mapas, Universidad Nacional Autónoma de México, Plaza y Valdés, pp. 89-109.

Mentz, M. (1982), México en el siglo XIX visto por los alemanes. México, Universidad Nacional Autónoma de México.

Milne-Edwards, Henri (1868), Mission Scientifique au Mexique et dans l'Amérique Centrale. Paris, Imprimerie Nationale.

Niox, G. L. (1873), Expédition du Mexique (1861-1867). Récit Politique et Militaire. Paris, Librairie Militaire de J. Dumanine.

Río, A. M. (1832), Elementos de Orictognosia, o del conocimiento de los fósiles, según el sistema de Bercelio; y según los principios de Abraham Göttlob Werner, con la sinonimia inglesa, alemana y francesa, para uso del Seminario Nacional de Minería. Parte práctica. Filadelfia, J. F. Hurtel Press.

Sainte-Claire Deville, C. (1865-1867), "Géologie et Minéralogie". En: Archives de la Commission Scientifique du Mexique, vol. I., Paris, Ministère de I'Instruction Publique, Imprimerie Impériale, pp. 37-48.

Tamayo, L. M. (2000), La frontera México-Estados Unidos. La conformación de un espacio durante el siglo XIX, Tesis inédita de Doctorado en Geografía. México, Facultad de Filosofía y Letras, Universidad Nacional Autónoma de México.

Tamayo, L. M. (2001), La geografía, arma cientifica para la defensa del territorio. México, Plaza y Valdés, Universidad Nacional Autónoma de México.

Torres O. (2012), "El viaje de William More Gabb a Baja California en 1867". En: L. F. Azuela y R. Vega y Ortega (coord.), Naturaleza y territorio en la ciencia mexicana del siglo XIX, México, Universidad Nacional Autónoma de México, pp. 107-135.

Vivien de Saint-Martin, L. (1865), "Rapport sur l'état actuel de la Géographie du Mexique et sur les études propres à perfectionner la carte du pays, par...". En: Archives de la Commission Scientifique du Mexique, vol. I., Paris, Ministère de I'Instruction Publique, Imprimerie Impériale, pp. 240-330.

Ward, H. G. (1981), México en 1827. México, Fondo de Cultura Económica. 